NenAd Ujević (Split)

\title{
INEQUALITIES OF OSTROWSKI-GRÜSS TYPE AND APPLICATIONS
}

Abstract. Some new inequalities of Ostrowski-Grüss type are derived. They are applied to the error analysis for some Gaussian and Gaussian-like quadrature formulas.

1. Introduction. In this paper we derive some new inequalities of Ostrowski-Grüss type. They are applied to the error analysis for some Gaussian and Gaussian-like quadrature formulas. We consider Gauss-Legendre, Chebyshev, Radau and Lobatto quadratures. A similar error analysis for rules of Newton-Cotes type can be found in the literature. In particular, the mid-point, trapezoid and Simpson rules have been investigated recently ([1], [2], [4], [8], [11]) with a view to obtaining bounds on the quadrature rule in terms of a variety of norms involving, at most, the first derivative.

The present work brings results for the above mentioned Gaussian and Gaussian-like quadrature rules giving explicit error bounds. We use Peano type kernels and an approach via inequalities. The error bounds are expressed in terms of first derivatives. The general approach used in the past required the assumption of bounded derivatives of degree higher than one. Our error bounds are in general (but not always) better than the Peano bounds.

We also consider composite quadrature rules. Our analysis allows the determination of a partition that would assure a prescribed error tolerance.

In Section 2 we establish some inequalities of Ostrowski-Grüss type. Such inequalities are considered in [2], [4], [8] and [11]. In Sections 3-6 we apply these inequalities to obtain explicit error bounds for the above mentioned quadrature rules. In Section 7 we give a general procedure for forming

2000 Mathematics Subject Classification: Primary 26D10, 41A55.

Key words and phrases: Ostrowski-Grüss inequalities, Gaussian quadrature, error analysis. 
composite quadrature rules and study some specific composite quadrature rules.

\section{Ostrowski-Grüss type inequalities}

Theorem 1. Let $I \subset \mathbb{R}$ be an open interval with $[-1,1] \subset I$. Let $f$ : $I \rightarrow \mathbb{R}$ be a differentiable function such that there exist constants $\gamma, \Gamma \in \mathbb{R}$ satisfying $\gamma \leq f^{\prime}(t) \leq \Gamma, t \in[-1,1]$. For any subdivision $-1=x_{0}<x_{1}<$ $\ldots<x_{n}<x_{n+1}=1$ of the interval $[-1,1]$ we have

$$
\begin{aligned}
\mid 2 \sum_{i=1}^{n} w_{i} f\left(x_{i}\right)- & {[f(1)-f(-1)] \sum_{i=1}^{n} w_{i} x_{i}-\left(\sum_{i=1}^{n} w_{i}\right) \int_{-1}^{1} f(t) d t \mid } \\
\leq & \frac{\Gamma-\gamma}{2} \sum_{j=0}^{n} \int_{x_{j}}^{x_{j+1}}\left|t \sum_{i=1}^{n} w_{i}-\sum_{i=1}^{n} w_{i} x_{i}+\sum_{i=1}^{n} w_{i} p_{i j}\right| d t
\end{aligned}
$$

where $w_{i} \geq 0$ are weights and

$$
p_{i j}= \begin{cases}1, & i>j \\ -1, & i \leq j\end{cases}
$$

for $i=1, \ldots, n, j=0,1, \ldots, n$.

Proof. We define

$$
K\left(x_{i}, t\right)= \begin{cases}t+1, & t \in\left[-1, x_{i}\right], \\ t-1, & t \in\left(x_{i}, 1\right],\end{cases}
$$

for $i=1, \ldots, n, x_{i} \in(-1,1)$. We have

$$
\begin{gathered}
\int_{-1}^{1} f^{\prime}(t) d t=f(1)-f(-1), \\
\int_{-1}^{1} K\left(x_{i}, t\right) d t=2 x_{i}, \quad i=1, \ldots, n .
\end{gathered}
$$

Integrating by parts, we obtain

$$
\int_{-1}^{1} K\left(x_{i}, t\right) f^{\prime}(t) d t=2 f\left(x_{i}\right)-\int_{-1}^{1} f(t) d t, \quad i=1, \ldots, n .
$$

From (2.4)-(2.6) it follows that

$$
\begin{aligned}
& \int_{-1}^{1} K\left(x_{i}, t\right) f^{\prime}(t) d t-\frac{1}{2} \int_{-1}^{1} K\left(x_{i}, t\right) d t \int_{-1}^{1} f^{\prime}(t) d t \\
& =2 f\left(x_{i}\right)-x_{i}[f(1)-f(-1)]-\int_{-1}^{1} f(t) d t, \quad i=1, \ldots, n
\end{aligned}
$$


It is not difficult to verify that

$$
\int_{-1}^{1}\left[K\left(x_{i}, t\right)-\frac{1}{2} \int_{-1}^{1} K\left(x_{i}, s\right) d s\right] d t=0, \quad i=1, \ldots, n .
$$

Hence

$$
\begin{aligned}
\int_{-1}^{1} K\left(x_{i}, t\right) f^{\prime}(t) d t & -\frac{1}{2} \int_{-1}^{1} K\left(x_{i}, t\right) d t \int_{-1}^{1} f^{\prime}(t) d t \\
& =\int_{-1}^{1} f^{\prime}(t)\left[K\left(x_{i}, t\right)-\frac{1}{2} \int_{-1}^{1} K\left(x_{i}, s\right) d s\right] d t \\
& =\int_{-1}^{1}\left[f^{\prime}(t)-C\right]\left[K\left(x_{i}, t\right)-\frac{1}{2} \int_{-1}^{1} K\left(x_{i}, s\right) d s\right] d t
\end{aligned}
$$

where $C \in \mathbb{R}$ is any constant.

From (2.7) and (2.9) we get

$$
\begin{aligned}
& 2 f\left(x_{i}\right)-x_{i}[f(1)-f(-1)]-\int_{-1}^{1} f(t) d t \\
& \quad=\int_{-1}^{1}\left[f^{\prime}(t)-C\right]\left[K\left(x_{i}, t\right)-\frac{1}{2} \int_{-1}^{1} K\left(x_{i}, s\right) d s\right] d t, \quad i=1, \ldots, n .
\end{aligned}
$$

We now multiply (2.10) by $w_{i} \in \mathbb{R}$ and sum to obtain

$$
\begin{aligned}
\sum_{i=1}^{n} w_{i}\left[2 f\left(x_{i}\right)-x_{i}[f(1)-f(-1)]-\int_{-1}^{1} f(t) d t\right] & \\
= & \int_{-1}^{1}\left[f^{\prime}(t)-C\right]\left\{\sum_{i=1}^{n} w_{i}\left[K\left(x_{i}, t\right)-\frac{1}{2} \int_{-1}^{1} K\left(x_{i}, s\right) d s\right]\right\} d t
\end{aligned}
$$

Clearly,

$$
\begin{aligned}
& \left|\int_{-1}^{1}\left[f^{\prime}(t)-C\right]\left\{\sum_{i=1}^{n} w_{i}\left[K\left(x_{i}, t\right)-\frac{1}{2} \int_{-1}^{1} K\left(x_{i}, s\right) d s\right]\right\} d t\right| \\
& \quad \leq \max _{t \in[-1,1]}\left|f^{\prime}(t)-C\right| \int_{-1}^{1}\left|\sum_{i=1}^{n} w_{i}\left[K\left(x_{i}, t\right)-\frac{1}{2} \int_{-1}^{1} K\left(x_{i}, s\right) d s\right]\right| d t
\end{aligned}
$$

For $C=(\Gamma+\gamma) / 2$ we get

$$
\max _{t \in[-1,1]}\left|f^{\prime}(t)-C\right| \leq \frac{\Gamma-\gamma}{2}
$$


We also have

$$
\begin{aligned}
\int_{-1}^{1} \mid \sum_{i=1}^{n} w_{i}\left[K\left(x_{i}, t\right)\right. & \left.-\frac{1}{2} \int_{-1}^{1} K\left(x_{i}, s\right) d s\right] \mid d t \\
= & \sum_{j=0}^{n} \int_{x_{j}}^{x_{j+1}}\left|t \sum_{i=1}^{n} w_{i}-\sum_{i=1}^{n} w_{i} x_{i}+\sum_{i=1}^{n} w_{i} p_{i j}\right| d t
\end{aligned}
$$

since $\sum_{i=1}^{n} w_{i} K\left(x_{i}, t\right)=t \sum_{i=1}^{n} w_{i}+\sum_{i=1}^{n} w_{i} p_{i j}$ and (2.5) holds.

From (2.10)-(2.14) we easily get (2.1).

REMARK 1. The above result is obtained by means of inequalities. It can also be derived using the Peano kernel theorem. In both cases the error bound, which is a Peano-like bound, is in general (but not always) better than the Peano bound. This is elaborated on below and holds for the rest of the results in this section.

We now wish to compare the estimate obtained with a classical one. For that purpose, we follow the presentation given in the survey article [5], using the quadrature formula given in Theorem 1. (See also the relevant references in $[5]$.

Let $R[f]=Q[f]-I[f]$, where $Q[f]$ is the quadrature formula given in Theorem 1,

$$
Q[f]=2 \sum_{i=1}^{n} w_{i} f\left(x_{i}\right)-[f(1)-f(-1)] \sum_{i=1}^{n} w_{i} x_{i}
$$

and

$$
I[f]=\left(\sum_{i=1}^{n} w_{i}\right) \int_{-1}^{1} f(t) d t
$$

We have $([5])$

$$
R[f]=\int_{-1}^{1} f^{\prime}(t) K_{1}(R, t) d t
$$

where $K_{1}(R, \cdot)$ is the so-called Peano kernel of $R$ of order one,

$$
K_{1}(R, t)=R\left[(\cdot-t)_{+}^{0}\right], \quad u_{+}^{0}= \begin{cases}1 & \text { for } u \geq 0 \\ 0 & \text { for } u<0 .\end{cases}
$$

Then the classical estimate of the remainder $R$ is

$$
|R[f]| \leq\left\|f^{\prime}\right\|_{\infty} \int_{-1}^{1}\left|K_{1}(R, t)\right| d t
$$

It is not difficult to find that on the interval $\left[x_{j}, x_{j+1}\right]$, the Peano kernel has 
the representation

$$
\begin{aligned}
K_{1}(t) & =2 \sum_{i=j+1}^{n} w_{i}-\sum_{i=1}^{n} w_{i} x_{i}-(1-t) \sum_{i=1}^{n} w_{i} \\
& =t \sum_{i=1}^{n} w_{i}-\sum_{i=1}^{n} w_{i} x_{i}+\sum_{i=1}^{n} w_{i} p_{i j},
\end{aligned}
$$

where we used the notations from Theorem 1. Thus,

$$
|R[f]| \leq\left\|f^{\prime}\right\|_{\infty} \sum_{j=0}^{n} \int_{x_{j}}^{x_{j+1}}\left|t \sum_{i=1}^{n} w_{i}-\sum_{i=1}^{n} w_{i} x_{i}+\sum_{i=1}^{n} w_{i} p_{i j}\right| d t
$$

or

$$
\begin{array}{r}
\left|2 \sum_{i=1}^{n} w_{i} f\left(x_{i}\right)-[f(1)-f(-1)] \sum_{i=1}^{n} w_{i} x_{i}-\left(\sum_{i=1}^{n} w_{i}\right) \int_{-1}^{1} f(t) d t\right| \\
\leq\left\|f^{\prime}\right\|_{\infty} \sum_{j=0}^{n} \int_{x_{j}}^{x_{j+1}}\left|t \sum_{i=1}^{n} w_{i}-\sum_{i=1}^{n} w_{i} x_{i}+\sum_{i=1}^{n} w_{i} p_{i j}\right| d t
\end{array}
$$

If we now choose

$$
\gamma=\inf _{t \in[-1,1]} f^{\prime}(t), \quad \Gamma=\sup _{t \in[-1,1]} f^{\prime}(t)
$$

then we obtain $(\Gamma-\gamma) / 2 \leq\left\|f^{\prime}\right\|_{\infty}$. Thus, the estimate (2.1) is better than (2.15). In fact, these two estimates are equal if and only if $\Gamma=-\gamma$. This last case is very rare in practice. On the other hand, the estimate (2.1) can be much better than (2.15): see Example 1 in Section 7.

Theorem 1 gives a general result. We now apply it to specific quadrature rules. For that purpose, we give the following five corollaries. Corollary 1 is meant for applications to Gauss-Legendre and Chebyshev quadrature rules. Corollaries 2 and 3 will be applied to Radau quadrature rules, and Corollaries 4 and 5 to Lobatto quadrature rules.

Corollary 1. Let the assumptions of Theorem 1 hold. If

$$
\sum_{i=1}^{n} w_{i}=1 \quad \text { and } \quad \sum_{i=1}^{n} w_{i} x_{i}=0
$$

then

$$
\left|2 \sum_{i=1}^{n} w_{i} f\left(x_{i}\right)-\int_{-1}^{1} f(t) d t\right| \leq \frac{\Gamma-\gamma}{2} \sum_{j=0}^{n} \int_{x_{j}}^{x_{j+1}}\left|t+\sum_{i=1}^{n} w_{i} p_{i j}\right| d t
$$

Proof. Substitute (2.16) in (2.1). 
Corollary 2. Under the assumptions of Theorem 1 we have

$$
\begin{aligned}
\mid 2 \sum_{i=0}^{n} w_{i} f\left(x_{i}\right) & -[f(1)-f(-1)] \sum_{i=0}^{n} w_{i} x_{i}-\left(\sum_{i=0}^{n} w_{i}\right) \int_{-1}^{1} f(t) d t \mid \\
\leq & \frac{\Gamma-\gamma}{2} \sum_{j=0}^{n} \int_{x_{j}}^{x_{j+1}}\left|t \sum_{i=0}^{n} w_{i}-\sum_{i=0}^{n} w_{i} x_{i}+\sum_{i=0}^{n} w_{i} p_{i j}\right| d t
\end{aligned}
$$

where $w_{0} \in \mathbb{R}$ and $p_{0 j}=-1, j=0,1, \ldots, n$.

Proof. We define

$$
K\left(x_{0}, t\right)=t-1,
$$

and let $K\left(x_{i}, t\right), i=1, \ldots, n$, be defined as in Theorem 1 . We now show that (2.5)-(2.7) hold for the additional function $K\left(x_{0}, t\right)$. We have

$$
\int_{-1}^{1} K\left(x_{0}, t\right) d t=-2=2 x_{0}
$$

since $x_{0}=-1$. Integrating by parts, we obtain

$$
\int_{-1}^{1} K\left(x_{0}, t\right) f^{\prime}(t) d t=2 f\left(x_{0}\right)-\int_{-1}^{1} f(t) d t .
$$

From (2.4), (2.20) and (2.21) we see that (2.7) holds for $i=0$.

We also have

$$
\int_{-1}^{1}\left[K\left(x_{0}, t\right)-\frac{1}{2} \int_{-1}^{1} K\left(x_{0}, s\right) d s\right] d t=0 .
$$

Now, it is not difficult to see that (2.9) holds for $i=0$.

From (2.20)-(2.22) and (2.4)-(2.9) it follows that

$$
\begin{aligned}
& \sum_{i=0}^{n} w_{i}\left[2 f\left(x_{i}\right)-x_{i}[f(1)-f(-1)]-\int_{-1}^{1} f(t) d t\right] \\
& =\int_{-1}^{1}\left[f^{\prime}(t)-C\right]\left\{\sum_{i=0}^{n} w_{i}\left[K\left(x_{i}, t\right)-\frac{1}{2} \int_{-1}^{1} K\left(x_{i}, s\right) d s\right]\right\} d t
\end{aligned}
$$

for $w_{i} \in \mathbb{R}, i=0,1, \ldots, n, C \in \mathbb{R}$.

If we now choose $C=(\Gamma+\gamma) / 2$ then we get (2.18).

COROLlary 3. Let the assumptions of Theorem 1 be satisfied. If

$$
\sum_{i=0}^{n} w_{i}=1 \quad \text { and } \quad \sum_{i=0}^{n} w_{i} x_{i}=0
$$


then

$$
\left|2 \sum_{i=0}^{n} w_{i} f\left(x_{i}\right)-\int_{-1}^{1} f(t) d t\right| \leq \frac{\Gamma-\gamma}{2} \sum_{j=0}^{n} \int_{x_{j}}^{x_{j+1}}\left|t+\sum_{i=0}^{n} w_{i} p_{i j}\right| d t .
$$

Proof. Substitute (2.24) in (2.18).

COROllary 4. Under the assumptions of Corollary 3 we have

$$
\begin{aligned}
\mid 2 \sum_{i=0}^{n+1} w_{i} f\left(x_{i}\right) & -[f(1)-f(-1)] \sum_{i=0}^{n+1} w_{i} x_{i}-\left(\sum_{i=0}^{n+1} w_{i}\right) \int_{-1}^{1} f(t) d t \mid \\
& \leq \frac{\Gamma-\gamma}{2} \sum_{j=0}^{n} \int_{x_{j}}^{x_{j+1}}\left|t \sum_{i=0}^{n+1} w_{i}-\sum_{i=0}^{n+1} w_{i} x_{i}+\sum_{i=0}^{n+1} w_{i} p_{i j}\right| d t
\end{aligned}
$$

where $w_{n+1} \in \mathbb{R}$ and $p_{n+1, j}=1, j=0,1, \ldots, n$.

Proof. We define

$$
K\left(x_{n+1}, t\right)=t+1
$$

and let $K\left(x_{i}, t\right), i=0,1, \ldots, n$, be defined as in Theorem 1 and Corollary 2 . We now show that $(2.5)-(2.7)$ hold for the additional function $K\left(x_{n+1}, t\right)$. We have

$$
\int_{-1}^{1} K\left(x_{n+1}, t\right) d t=2=2 x_{n+1}
$$

since $x_{n+1}=1$. Integrating by parts, we obtain

$$
\int_{-1}^{1} K\left(x_{n+1}, t\right) f^{\prime}(t) d t=2 f\left(x_{n+1}\right)-\int_{-1}^{1} f(t) d t .
$$

From (2.4), (2.28) and (2.29) we see that (2.7) holds for $i=n+1$.

We also have

$$
\int_{-1}^{1}\left[K\left(x_{n+1}, t\right)-\frac{1}{2} \int_{-1}^{1} K\left(x_{n+1}, s\right) d s\right] d t=0
$$

Now, it is not difficult to see that (2.9) holds for $i=n+1$.

From (2.28)-(2.30), (2.20)-(2.22) and (2.4)-(2.9) it follows that

$$
\begin{aligned}
& \sum_{i=0}^{n+1} w_{i}\left[2 f\left(x_{i}\right)-x_{i}[f(1)-f(-1)]-\int_{-1}^{1} f(t) d t\right] \\
& =\int_{-1}^{1}\left[f^{\prime}(t)-C\right]\left\{\sum_{i=0}^{n+1} w_{i}\left[K\left(x_{i}, t\right)-\frac{1}{2} \int_{-1}^{1} K\left(x_{i}, s\right) d s\right]\right\} d t
\end{aligned}
$$

for $w_{i} \in \mathbb{R}, i=0,1, \ldots, n+1, C \in \mathbb{R}$.

If we now choose $C=(\Gamma+\gamma) / 2$ then we get $(2.26)$. 
Corollary 5. Let the assumptions of Corollary 4 be satisfied. If

$$
\sum_{i=0}^{n+1} w_{i}=1 \quad \text { and } \quad \sum_{i=0}^{n+1} w_{i} x_{i}=0
$$

then

$$
\left|2 \sum_{i=0}^{n+1} w_{i} f\left(x_{i}\right)-\int_{-1}^{1} f(t) d t\right| \leq \frac{\Gamma-\gamma}{2} \sum_{j=0}^{n} \int_{x_{j}}^{x_{j+1}}\left|t+\sum_{i=0}^{n+1} w_{i} p_{i j}\right| d t
$$

Proof. Substitute (2.32) in (2.26).

3. Gauss-Legendre quadrature. We have

$$
\int_{-1}^{1} f(t) d t=2 \sum_{i=1}^{n} w_{i} f\left(x_{i}\right)+E_{n}(f), \quad x_{i} \in(-1,1)
$$

where

$$
E_{n}(f)=\frac{2^{2 n+1}(n !)^{4}}{(2 n+1)[(2 n) !]^{3}} f^{(2 n)}(\xi), \quad \xi \in(-1,1) .
$$

We can find $x_{i}, w_{i}$ in [12]. We apply Corollary 1 to obtain new error bounds for this type of quadrature rules.

CASE $n=2$. We have

$$
x_{1}=-\sqrt{3} / 3, \quad x_{2}=\sqrt{3} / 3, \quad w_{1}=1 / 2, \quad w_{2}=1 / 2 .
$$

It is not difficult to verify that the conditions (2.16) are satisfied. We have

$$
\int_{-1}^{x_{1}}|t+1| d t+\int_{x_{1}}^{x_{2}}|t| d t+\int_{x_{2}}^{1}|t-1| d t=\frac{5}{3}-\frac{2}{3} \sqrt{3} .
$$

Hence,

$$
\left|2 \sum_{i=1}^{2} w_{i} f\left(x_{i}\right)-\int_{-1}^{1} f(t) d t\right| \leq \frac{\Gamma-\gamma}{6}(5-2 \sqrt{3}) .
$$

CASE $n=3$. We now have

$$
\begin{aligned}
& x_{1}=-\sqrt{15} / 5, \quad x_{2}=0, \quad x_{3}=\sqrt{15} / 5, \\
& w_{1}=5 / 18, \quad w_{2}=4 / 9, \quad w_{3}=5 / 18 .
\end{aligned}
$$

The conditions (2.16) are satisfied so that we can apply Corollary 1 . We have

$$
\int_{-1}^{x_{1}}|t+1| d t+\int_{x_{1}}^{x_{2}}\left|t+\frac{4}{9}\right| d t+\int_{x_{2}}^{x_{3}}\left|t-\frac{4}{9}\right| d t+\int_{x_{2}}^{1}|t-1| d t=\frac{1051}{405}-\frac{26}{45} \sqrt{15}
$$


Hence,

$$
\left|2 \sum_{i=1}^{3} w_{i} f\left(x_{i}\right)-\int_{-1}^{1} f(t) d t\right| \leq \frac{\Gamma-\gamma}{2}\left(\frac{1051}{405}-\frac{26}{45} \sqrt{15}\right)
$$

4. Chebyshev quadrature. We have

$$
\int_{-1}^{1} f(t) d t=\frac{2}{n} \sum_{i=1}^{n} f\left(x_{i}\right)+E_{n}(f), \quad x_{i} \in(-1,1)
$$

where

$$
E_{n}(f)= \begin{cases}\frac{c_{n}}{(n+1) !} f^{(n+1)}(\xi), & n \text { odd } \\ \frac{c_{n}}{(n+2) !} f^{(n+2)}(\xi), & n \text { even }\end{cases}
$$

where $c_{n}=$ const $\left(c_{2}=2 / 3, c_{3}=8 / 45\right), \xi \in(-1,1)$.

We can find $x_{i}$ in [12]. We again apply Corollary 1 . The case $n=2$ is the same as for the Legendre-Gauss quadrature.

CASE $n=3$. We have

$$
\begin{array}{lll}
x_{1}=-\sqrt{2} / 2, & x_{2}=0, & x_{3}=\sqrt{2} / 2, \\
w_{1}=1 / 3, & w_{2}=1 / 3, & w_{3}=1 / 3 .
\end{array}
$$

The conditions (2.16) are satisfied so that we can apply Corollary 1 . We have

$$
\int_{-1}^{x_{1}}|t+1| d t+\int_{x_{1}}^{x_{2}}\left|t+\frac{1}{3}\right| d t+\int_{x_{2}}^{x_{3}}\left|t-\frac{1}{3}\right| d t+\int_{x_{3}}^{1}|t-1| d t=\frac{20}{9}-\frac{4}{3} \sqrt{2}
$$

Hence,

$$
\left|2 \sum_{i=1}^{3} w_{i} f\left(x_{i}\right)-\int_{-1}^{1} f(t) d t\right| \leq \frac{\Gamma-\gamma}{18}(20-12 \sqrt{2}) .
$$

CASE $n=4$. We have

$$
\begin{array}{llll}
x_{1}=-\sqrt{\frac{\sqrt{5}+2}{3 \sqrt{5}},} & x_{2}=-\sqrt{\frac{\sqrt{5}-2}{3 \sqrt{5}},} & x_{3}=\sqrt{\frac{\sqrt{5}-2}{3 \sqrt{5}}}, & x_{4}=\sqrt{\frac{\sqrt{5}+2}{3 \sqrt{5}}} \\
w_{1}=\frac{1}{4}, & w_{2}=\frac{1}{4}, & w_{3}=\frac{1}{4}, & w_{4}=\frac{1}{4} .
\end{array}
$$

The conditions (2.16) are satisfied. We have

$$
\begin{aligned}
\int_{-1}^{x_{1}}|t+1| d t+\int_{x_{1}}^{x_{2}} \mid t+ & \frac{1}{2}\left|d t+\int_{x_{2}}^{x_{3}}\right| t\left|d t+\int_{x_{3}}^{x_{4}}\right| t-\frac{1}{2}\left|d t+\int_{x_{4}}^{1}\right| t-1 \mid d t \\
& =-\frac{1}{5} \sqrt{15(5+2 \sqrt{5})}+\frac{17}{6}-\frac{1}{15} \sqrt{15(5-2 \sqrt{5})}
\end{aligned}
$$


Hence,

$$
\begin{aligned}
\mid 2 \sum_{i=1}^{4} w_{i} f\left(x_{i}\right) & -\int_{-1}^{1} f(t) d t \mid \\
\leq & \frac{\Gamma-\gamma}{2}\left(-\frac{1}{5} \sqrt{15(5+2 \sqrt{5})}+\frac{17}{6}-\frac{1}{15} \sqrt{15(5-2 \sqrt{5})}\right) .
\end{aligned}
$$

5. Radau quadrature. We have

$$
\int_{-1}^{1} f(t) d t=2 w_{0} f(-1)+2 \sum_{i=1}^{n} w_{i} f\left(x_{i}\right)+E_{n}(f), \quad x_{i} \in(-1,1),
$$

where

$$
E_{n}(f)=\frac{2^{2 n-1} n[(n-1) !]^{4}}{[(2 n-1) !]^{3}} f^{(2 n-1)}(\xi), \quad \xi \in(-1,1) .
$$

We now apply Corollary 3 to obtain some new estimates of the remainders. We can find $x_{i}, w_{i}$ in [12].

CASE $n=1$. We have

$$
x_{0}=-1, \quad x_{1}=1 / 3, \quad w_{0}=1 / 4, \quad w_{1}=3 / 4 .
$$

The conditions (2.24) are satisfied. Thus we can apply Corollary 3 . We have

$$
\int_{-1}^{x_{1}}\left|t+\frac{1}{2}\right| d t+\int_{x_{1}}^{1}|t-1| d t=\frac{25}{36}
$$

Hence,

$$
\left|2 \sum_{i=0}^{1} w_{i} f\left(x_{i}\right)-\int_{-1}^{1} f(t) d t\right| \leq \frac{25}{72}(\Gamma-\gamma)
$$

CASE $n=2$. We have

$$
\begin{array}{lll}
x_{0}=-1, & x_{1}=\frac{1-\sqrt{6}}{5}, & x_{2}=\frac{1+\sqrt{6}}{5}, \\
w_{0}=\frac{1}{9}, & w_{1}=\frac{16+\sqrt{6}}{36}, & w_{2}=\frac{16-\sqrt{6}}{36} .
\end{array}
$$

The conditions (2.24) hold. We have

$$
\int_{-1}^{x_{1}}\left|t+\frac{7}{9}\right| d t+\int_{x_{1}}^{x_{2}}\left|t-\frac{2+\sqrt{6}}{18}\right| d t+\int_{x_{2}}^{1}|t-1| d t=\frac{5383}{4050}-\frac{148}{405} \sqrt{6} .
$$

Hence,

$$
\left|2 \sum_{i=0}^{2} w_{i} f\left(x_{i}\right)-\int_{-1}^{1} f(t) d t\right| \leq \frac{\Gamma-\gamma}{2}\left(\frac{5383}{4050}-\frac{148}{405} \sqrt{6}\right)
$$


6. Lobatto quadrature. We have

$\int_{-1}^{1} f(t) d t=2 w_{0} f(-1)+2 w_{n+1} f(1)+2 \sum_{i=1}^{n} w_{i} f\left(x_{i}\right)+E_{n}(f), \quad x_{i} \in(-1,1)$, where

$$
E_{n}(f)=-\frac{n(n-1)^{3} 2^{2 n-1}[(n-2) !]^{4}}{(2 n-1)[(2 n-2) !]^{3}} f^{(2 n-2)}(\xi), \quad \xi \in(-1,1) .
$$

We can find $x_{i}, w_{i}$ in [12]. We apply Corollary 5 .

CASE $n=1$. We have

$$
\begin{array}{lll}
x_{0}=-1, & x_{1}=0, & x_{2}=1, \\
w_{0}=1 / 6, & w_{1}=2 / 3, & w_{3}=1 / 6 .
\end{array}
$$

The conditions (2.32) are satisfied. We have

$$
\int_{-1}^{x_{1}}\left|t+\frac{2}{3}\right| d t+\int_{x_{1}}^{1}\left|t-\frac{2}{3}\right| d t=\frac{5}{9}
$$

Hence,

$$
\left|2 \sum_{i=0}^{2} w_{i} f\left(x_{i}\right)-\int_{-1}^{1} f(t) d t\right| \leq \frac{5}{18}(\Gamma-\gamma)
$$

CASE $n=2$. We have

$$
\begin{array}{llll}
x_{0}=-1, & x_{1}=-\sqrt{5} / 5, & x_{2}=\sqrt{5} / 5, & x_{3}=1, \\
w_{0}=1 / 12, & w_{1}=5 / 12, & w_{2}=5 / 12, & w_{3}=1 / 12 .
\end{array}
$$

The conditions (2.32) are satisfied. We have

$$
\int_{-1}^{x_{1}}\left|t+\frac{5}{6}\right| d t+\int_{x_{1}}^{x_{2}}|t| d t+\int_{x_{2}}^{1}\left|t-\frac{5}{6}\right| d t=\frac{101}{90}-\frac{1}{3} \sqrt{5}
$$

Hence,

$$
\left|2 \sum_{i=0}^{3} w_{i} f\left(x_{i}\right)-\int_{-1}^{1} f(t) d t\right| \leq \frac{\Gamma-\gamma}{2}\left(\frac{101}{90}-\frac{1}{3} \sqrt{5}\right) .
$$

CASE $n=3$. We have

$$
\begin{array}{lllll}
x_{0}=-1, & x_{1}=-\sqrt{21} / 7, & x_{2}=0, & x_{3}=\sqrt{21} / 7, & x_{4}=1, \\
w_{0}=1 / 20, & w_{1}=49 / 180, & w_{2}=16 / 45, & w_{3}=49 / 180, & w_{4}=1 / 20 .
\end{array}
$$

The conditions (2.32) hold. We have

$$
\begin{aligned}
\int_{-1}^{x_{1}}\left|t+\frac{9}{10}\right| d t+\int_{x_{1}}^{x_{2}}\left|t+\frac{16}{45}\right| d t+\int_{x_{3}}^{x_{4}}\left|t-\frac{16}{45}\right| d t+\int_{x_{4}}^{1}\left|t-\frac{9}{10}\right| \\
=\frac{10943}{5670}-\frac{113}{315} \sqrt{21}
\end{aligned}
$$


Hence,

$$
\left|2 \sum_{i=0}^{4} w_{i} f\left(x_{i}\right)-\int_{-1}^{1} f(t) d t\right| \leq \frac{\Gamma-\gamma}{2}\left(\frac{10943}{5670}-\frac{113}{315} \sqrt{21}\right) .
$$

7. Composite quadrature rules. With the aid of the quadrature formulas given in Corollaries 1,3 and 5 with $n$ (resp. $n+1, n+2$ ) nodes in the interval $[-1,1]$, which will be called canonical, we can form composite quadrature formulas on an arbitrary interval $[a, b]$. To this end, divide $[a, b]$ into $N$ equal subintervals $\left[x_{k}, x_{k+1}\right], x_{k}=a+k(b-a) / N, k=0,1, \ldots, N-1$ and $x_{N}=b$. On each subinterval $\left[x_{k}, x_{k+1}\right]$ we specify $n$ (resp. $\left.n+1, n+2\right)$ nodes

$$
x_{k_{j}}=\frac{x_{k}+x_{k+1}}{2}+y_{j} \frac{b-a}{2 N}, \quad j=l, l+1, \ldots, m,
$$

where $y_{j}$ are the nodes of the corresponding canonical formulas. We have: $l=1, m=n$ for the Gauss-Legendre and Chebyshev quadrature, $l=0$, $m=n$ for the Radau quadrature, and $l=0, m=n+1$ for the Lobatto quadrature. Hence, we have the quadrature formula

$$
\int_{x_{k}}^{x_{k+1}} f(t) d t=\frac{b-a}{N} \sum_{j=l}^{m} w_{j} f\left(x_{k_{j}}\right)+R_{k}(f)
$$

where $w_{j}$ are the weights of the corresponding canonical formulas. Summing (7.2) over $k$ from 0 to $N-1$, we obtain the composite quadrature formula

$$
\int_{a}^{b} f(t) d t=\frac{b-a}{N} \sum_{j=l}^{m} w_{j} \sum_{k=0}^{N-1} f\left(x_{k_{j}}\right)+R(f) .
$$

We give explicit results only for the cases $l=1, m=2$ (Gauss-Legendre and Chebyshev quadrature), $l=0, m=1$ (Radau quadrature) and $l=0$, $m=2$ (Lobatto quadrature); in a similar way we can obtain composite quadrature formulas for other cases. (The general procedure of forming these formulas is described above.)

TheOREM 2. Under the notations defined above let $I \subset \mathbb{R}$ be an open interval with $[a, b] \subset I$. Let $f: I \rightarrow R$ be a differentiable function such that there exist constants $\gamma, \Gamma \in \mathbb{R}$ satisfying $\gamma \leq f^{\prime}(t) \leq \Gamma, t \in[a, b]$. Then

$$
\left|\int_{a}^{b} f(t) d t-\frac{h}{2} \sum_{k=0}^{N-1}\left[f\left(x_{k 1}\right)+f\left(x_{k 2}\right)\right]\right| \leq \frac{\Gamma-\gamma}{24 N}(5-2 \sqrt{3})(b-a)^{2}
$$

where

$$
x_{k 1}=\frac{x_{k}+x_{k+1}}{2}-\frac{\sqrt{3}}{6} h, \quad x_{k 2}=\frac{x_{k}+x_{k+1}}{2}+\frac{\sqrt{3}}{6} h
$$

and $h=(b-a) / N$. 
Proof. From (7.1), (7.2) and the case $n=2$ of the Gauss-Legendre quadrature we have

$$
\int_{x_{k}}^{x_{k+1}} f(t) d t=\frac{h}{2}\left[f\left(x_{k 1}\right)+f\left(x_{k 2}\right)\right]+R_{k}(f)
$$

where

$$
\left|R_{k}(f)\right| \leq \frac{\Gamma-\gamma}{24}(5-2 \sqrt{3}) h^{2}
$$

Summing (7.5) over $k$ from 0 to $N-1$ yields

$$
\int_{a}^{b} f(t) d t=\frac{h}{2} \sum_{k=0}^{N-1}\left[f\left(x_{k 1}\right)+f\left(x_{k 2}\right)\right]+\sum_{k=0}^{N-1} R_{k}(f) .
$$

From (7.7) and (7.6) we easily find that (7.4) holds.

EXAmple 1. Consider the integral $\int_{0}^{x} \sin \left(t+t^{2}\right) d t$. Suppose that we have to calculate it with error tolerance $10^{-5}$ for $x=0.1$ using the above composite quadrature rule. The classical estimate for the rule (see Section 2) is given by

$$
\left|\int_{a}^{b} f(t) d t-\frac{h}{2} \sum_{k=0}^{N-1}\left[f\left(x_{k 1}\right)+f\left(x_{k 2}\right)\right]\right| \leq \frac{\left\|f^{\prime}\right\|_{\infty}}{12 N}(5-2 \sqrt{3})(b-a)^{2} .
$$

Since $f^{\prime}(t)=(1+2 t) \cos \left(t+t^{2}\right)$ we have $\left\|f^{\prime}\right\|_{\infty}=1.192747318=\Gamma$ and $\gamma=1$. Using (7.8) we find that $N \geq 153$ while (7.4) yields $N \geq 13$. Hence, the estimate (7.8) tells us that we have to perform 306 function evaluations to obtain the given tolerance, while by (7.4) only 26 function evaluations are required. Recall that function evaluations are generally considered the computationally most expensive part of quadrature algorithms.

TheOREM 3. Under the assumptions of Theorem 2 we have

$$
\left|\int_{a}^{b} f(t) d t-h \sum_{k=0}^{N-1}\left[\frac{1}{4} f\left(x_{k 0}\right)+\frac{3}{4} f\left(x_{k 1}\right)\right]\right| \leq 25 \frac{\Gamma-\gamma}{288 N}(b-a)^{2},
$$

where

$$
x_{k 0}=x_{k}, \quad x_{k 1}=\frac{x_{k}+x_{k+1}}{2}-\frac{h}{6} .
$$

Proof. From (7.1), (7.2) and the case $n=1$ of the Radau quadrature we have

$$
\int_{x_{k}}^{x_{k+1}} f(t) d t=h\left[\frac{1}{4} f\left(x_{k 0}\right)+\frac{3}{4} f\left(x_{k 1}\right)\right]+R_{k}(f),
$$

where

$$
\left|R_{k}(f)\right| \leq 25 \frac{\Gamma-\gamma}{288} h^{2}
$$


Summing (7.10) over $k$ from 0 to $N-1$ yields

$$
\int_{a}^{b} f(t) d t=h \sum_{k=0}^{N-1}\left[\frac{1}{4} f\left(x_{k 0}\right)+\frac{3}{4} f\left(x_{k 1}\right)\right]+\sum_{k=0}^{N-1} R_{k}(f) .
$$

From (7.12) and (7.11) we easily find that (7.9) holds.

TheOREM 4. Under the assumptions of Theorem 2 we have

$$
\begin{aligned}
\mid \int_{a}^{b} f(t) d t-h \sum_{k=0}^{N-1}\left[\frac{1}{6} f\left(x_{k 0}\right)+\frac{2}{3} f\left(x_{k 1}\right)+\right. & \left.\frac{1}{6} f\left(x_{k 2}\right)\right] \mid \\
& \leq 5 \frac{\Gamma-\gamma}{72 N}(b-a)^{2}
\end{aligned}
$$

where

$$
x_{k 0}=x_{k}, \quad x_{k 1}=\frac{x_{k}+x_{k+1}}{2}, \quad x_{k 2}=x_{k+1} .
$$

Proof. From (7.1), (7.2) and the case $n=2$ of the Lobatto quadrature we have

$$
\int_{x_{k}}^{x_{k+1}} f(t) d t=h\left[\frac{1}{6} f\left(x_{k 0}\right)+\frac{2}{3} f\left(x_{k 1}\right)+\frac{1}{6} f\left(x_{k 2}\right)\right]+R_{k}(f),
$$

where

$$
\left|R_{k}(f)\right| \leq 5 \frac{\Gamma-\gamma}{72} h^{2}
$$

Summing (7.14) over $k$ from 0 to $N-1$ gives

$$
\int_{a}^{b} f(t) d t=h \sum_{k=0}^{N-1}\left[\frac{1}{6} f\left(x_{k 0}\right)+\frac{2}{3} f\left(x_{k 1}\right)+\frac{1}{6} f\left(x_{k 2}\right)\right]+\sum_{k=0}^{N-1} R_{k}(f) .
$$

From (7.16) and (7.15) we easily find that (7.13) holds.

REMARK 2. Note that the quadrature formula given in Theorem 4 is the well-known Simpson quadrature formula. It can be rewritten in the form

$$
Q[f]=\frac{h}{6}\left[f\left(x_{0}\right)+f\left(x_{N}\right)+2 \sum_{k=1}^{N-1} f\left(x_{2 k}\right)+4 \sum_{k=0}^{N-1} f\left(x_{2 k+1}\right)\right] .
$$

Note also that the classical error bound for this formula is given by $\frac{M_{4}}{180} h^{4}(b-a)$, where $M_{4}=\sup _{t \in[a, b]}\left|f^{(4)}(t)\right|$. Hence, if $f$ does not belong to $C^{4}(a, b)$ then we cannot apply the classical formula, i.e. we cannot determine (estimate) the error.

\section{References}

[1] G. A. Anastassiou, Ostrowski type inequalities, Proc. Amer. Math. Soc. 123 (1995), 3775-3781. 
[2] P. Cerone, S. S. Dragomir and J. Roumeliotis, An inequality of Ostrowski-Grüss type for twice differentiable mappings and applications in numerical integration, Kyungpook Math. J. 39 (1999), 331-341.

[3] P. J. Davis and P. Rabinowitz, Methods of Numerical Integration, Academic Press, New York, 1975.

[4] S. S. Dragomir and S. Wang, An inequality of Ostrowski-Grüss type and its applications to the estimation of error bounds for some special means and for some numerical quadrature rules, Comput. Math. Appl. 33 (1997), 16-20.

[5] S. Ehrich, Stopping functionals for Gaussian quadrature formulas, J. Comput. Appl. Math. 127 (2001), 153-171.

[6] A. M. Fink, Bound on the deviation of a function from its averages, Czechoslovak Math. J. 42 (117) (1992), 289-310.

[7] A. Ghizzetti and A. Ossicini, Quadrature Formulae, Birkhäuser, Basel, 1970.

[8] M. Matić, J. Pečarić and N. Ujević, Improvement and further generalization of some inequalities of Ostrowski-Grüss type, Comput. Math. Appl. 39 (2000), 161-175.

[9] D. S. Mitrinović, J. E. Pečarić and A. M. Fink, Classical and New Inequalities in Analysis, Kluwer, Dordrecht, 1993.

[10] - , - - - Inequalities Involving Functions and Their Integrals and Derivatives, Kluwer, Dordrecht, 1991.

[11] C. E. M. Pearce, J. Pečarić, N. Ujević and S. Varošanec, Generalizations of some inequalities of Ostrowski-Grüss type, Math. Inequal. Appl. 3 (2000), 25-34.

[12] E. W. Weisstein, CRC Concise Encyclopedia of Mathematics, CRC, 1999.

Department of Mathematics

University of Split

Teslina 12/III

21000 Split, Croatia

E-mail: ujevic@pmfst.hr

Received on 11.4.2002;

revised version on 10.7 .2002 